\title{
Alteration of Gut Microbiota and Its Impact on Immune Response in Patients with Chronic HBV Infection: A Review
}

\author{
Yeshimebet Kassa' \\ Yihenew Million iD ${ }^{2}$ \\ Alemu Gedefiel \\ Feleke Moges iD ${ }^{2}$ \\ 'Department of Medical Laboratory \\ Sciences, College of Medicine and Health \\ Science, Wollo University, Dessie, \\ Ethiopia; ${ }^{2}$ Department of Medical \\ Microbiology, School of Biomedical and \\ Laboratory Sciences, College of Medicine \\ and Health Sciences, University of \\ Gondar, Gondar, Ethiopia
}

Correspondence: Yeshimebet Kassa Department of Medical Laboratory Science, College of Medicine and Health Sciences, Wollo University, PO.BOX:

I 145, Dessie, Ethiopia

Tel $+25 \mathrm{I}-915778098$

Fax +25I 333II5250

Email kassayeshimebet@gmail.com

\begin{abstract}
Chronic hepatitis B virus infection is a source of substantial global health problems, particularly in economically underdeveloped and/or developing countries. It is the primary cause of severe liver disorders such as liver fibrosis, cirrhosis, and hepatocellular carcinoma. The liver is connected by the bile duct to the small intestine that carries bile produced in the liver to the intestine. The liver is the initial organ exposed to materials originating from the gut including dietary compounds, bacteria, and their products. Human intestines harbor a wide diversity of the community of microbes which are collectively termed as gut microbiota. In chronic infection with the hepatitis B virus, microbial alteration of the gut is a source of systemic immune activation. Besides, gut permeability is altered in hepatitis B virus-infected patients with an increased bacterial translocation and endotoxin load in the portal vein that caused toll-like receptor activation in the liver, which facilitates immune-mediated liver injury. Toll-like receptors further triggered the host-wide inflammatory response by inducing signaling cascades such as nuclear factor-kappa B-linked pathways and by accelerating cytokine secretion like tumor necrosis factor-alpha, which evokes chronic inflammation and leads to liver lesion formation, fibrosis progression, and cirrhosis and hepatocellular carcinoma development. In conclusion, changes in intestinal flora play an important role in encouraging the production of chronic infection with the hepatitis B virus. Therefore, careful attention should be given to the maintenance of intestinal microecology of patients which can provide a sound foundation for the treatment of chronic infection with the hepatitis B virus.
\end{abstract}

Keywords: hepatitis B virus, gut microbiota, immune response

\section{Introduction}

Chronic hepatitis $\mathrm{B}(\mathrm{CHB})$ infection is a global epidemic disease that results from hepatitis $\mathrm{B}$ virus (HBV) infection and may progress to severe liver disorders, including liver fibrosis, cirrhosis, and hepatocellular carcinoma. ${ }^{1}$ Hepatitis B virus (HBV) is a noncytopathic, hepatotropic virus of the family Hepadna viridae; it is an enveloped virus with circular partially double-stranded DNA representing the highly compact organization. $\mathrm{HBV}$ is the smallest known DNA virus, having a spherical shape of approximately $42 \mathrm{~nm}$ in diameter and a genomic length of about 3200 base pairs. $^{2,3}$

Hepatitis $\mathrm{B}$ virus has four overlapping open reading frames: preC/C that encodes for e antigen $(\mathrm{HBeAg})$ and core protein $(\mathrm{HBcAg}), \mathrm{P}$ for polymerase (reverse transcriptase), $\mathrm{S}$ for surface proteins, and $\mathrm{X}$ for a transcriptional trans-activator protein. 
HBV is replicated by reverse transcription of pre-genomic RNA, which is transcribed from the covalently closed-circular form of HBV DNA in the hepatocyte nucleus by cellular RNA polymerase II. Sequence heterogeneity is a characteristic of HBV since POL cannot proofread. ${ }^{4}$ According to phylogenetic analysis, HBV can be classified into ten, A-J, based upon an inter-group divergence., ${ }^{4,5}$

HBV surface antigen (HBsAg) indicates an acute or chronic infection, and it is commonly regarded as a serological marker for HBV infection. Infection is considered chronic if it persists for greater than 6 months. HBsAg disappearance and HBV surface antibody (HBsAb) emergence indicate the recovery from infection and the development of $\mathrm{HBV}$ immunity. ${ }^{6} \mathrm{HBV}$ core antigen ( $\mathrm{HBcAg}$ ) consists of the core particle of the virus that is surrounded by HBsAg. HBV e antigen ( $\mathrm{HBe} A g$ ), the soluble secretory form of $\mathrm{HBcAg}$, serves as an important serological marker indicating viral replication. $\mathrm{HBeAg}$ to e antibody (HBeAb) conversion suggests host immune control and low replication of $\mathrm{HBV}^{7}$ Adult infections have a relatively low chronicity rate (around 5\%); typically, neonatal infections have a high persistence rate. ${ }^{8-10}$ Chronic infections are often asymptomatic, but carriers of HBV are at risk of developing lifethreatening cirrhosis and later on hepatic carcinoma. ${ }^{11}$

Standard treatment regimens with pegylated interferon (IFN)- $\alpha$ and nucleoside/nucleotide analogs are used to treat chronic hepatitis $\mathrm{B}$, but unfortunately, it neglects the role of gut microbiota (GM) balance in patients with chronic hepatitis B virus-infected. Increasing pieces of evidence have shown that gut microbiota plays an important role in the development of liver disease, pathogenesis, and response to treatment. ${ }^{1,12}$

Gut microbial alteration is a cause of systemic immune activation in chronic HBV infection. Translocation of gut microbial products, bacterial peptidoglycan, flagellin, and metabolic by-products has been suggested to exacerbate the clinical course of patients with chronic HBV infection, and intestinal dysfunction in liver cirrhosis is well known. ${ }^{13,14}$ A better understanding of the pathophysiological connection between gut microbiota alteration and its impact on the hepatic immune response is crucial for the development of new therapy to treat chronic hepatitis B virus infection.

\section{Host Immunity and Chronic HBV Infection}

Chronic HBV infection is one of the major global health problems, especially in economically underdeveloped or developing countries. It is the main cause of severe liver disorders, like liver fibrosis, cirrhosis, and hepatocellular carcinoma. According to World Health Organization (WHO) report, there are approximately 257 million people worldwide with chronic HBV infection, 887,000 of whom die of complications caused by chronic HBV infection. ${ }^{15}$

HBV immune response generally involves innate immunity and adaptive immunity; however, the successful innate immune response can not only directly eradicate the virus but can also have a substantial impact on unique immunity to HBV. ${ }^{16}$ The primary response to HBV infection is initiated by the innate immune system. Immunocytes of the innate immune system, including monocytes, macrophages, dendritic cells (DCs), neutrophils, natural killer cells (NK), and natural killer T cells (NKT), orchestrate innate immune systems and activate adaptive immune responses. ${ }^{17}$ The first important role of the immune response is appropriate sensing and recognition of invading microbes by Toll-like receptors (TLRs). ${ }^{18}$

Toll-like receptors are a group of highly conserved molecules that play a key role in recognizing pathogenassociated molecular patterns (PAMPs) and triggering innate immune responses to infectious agents. ${ }^{19}$ TLRs play a significant role in interferon and pro-inflammatory cytokine synthesis as well as immune cell mobilization to suppress viral replication. ${ }^{16,20}$

In the early stage of infection, toll-like receptors, especially TLR3 to detect intracellular HBV virions and TLR2/ 4 that bind to extracellular HBV components, are likely to be involved. Against HBV invasion, dendritic cells, Kupffer cells, NK cells, and NKT cells cooperate. Then, HBV-specific T-cells of CD4+ and CD8+ T-cells, the major HBV clearance effectors, induce IFN- $\gamma$ production and proliferation of $\mathrm{CD} 8+\mathrm{T}$ cells, as well as the production of multiple HBV antibodies and other cytokines. ${ }^{17}$ Generally, the key mechanisms by which HBV replication is suppressed in liver cells are usually the activation of cell signaling pathways by TLRs and the release of antiviral cytokines. $^{19}$

Generally, host immune systems play an important role in the outcome of HBV infection. However, the immunological mechanisms by which HBV establishes and maintains chronic infection, cirrhosis, and hepatocellular carcinoma are still under debate. ${ }^{17}$ However, the immune responses to $\mathrm{HBV}$ antigens are responsible for both viral clearances during acute infection and disease pathogenesis. The cause of liver injury is currently thought not to be the replication of $\mathrm{HBV}$ in liver cells, but rather the 
immune response caused by HBV. Chronic HBV-infected patients fail to develop $\mathrm{HBV}$-specific immune responses; for example, inappropriate, excessive, and nonspecific effector responses might be involved in persistent $\mathrm{HBV}$ replication and pathogenesis of $\mathrm{HBV}$-associated liver inflammation. $^{21}$

Another study has shown that HBeAg in patients with chronic HBV infection can minimize TLR2 expression in liver Kupffer cells and mononuclear cells. ${ }^{18}$ A recent study also found that the viral load of HBV DNA is negatively associated with the expression of TLR7 in biopsy samples, suggesting that HBV can inhibit TLR7 expression in liver cells. $^{22}$ Obviously, TLR-mediated signaling pathways induce immune responses to $\mathrm{HBV}$ infection, but the expression of TLRs on the immune cells is down-regulated by HBV itself. A significant number of studies on the gutliver axis have currently shown that improvements in intestinal microbiota play a key role in the induction and development of chronic hepatitis B infection. ${ }^{23,24}$

The involvement of gut microbiota (GM) in HBV clearance was demonstrated in animal models. The research found that, after six weeks of infection, adult mice with mature GM managed to clear up HBV, which is the opposite of young mice without GM who remained HBV positive. The fact that adult mice did not clear HBV after antibiotic sterilization of the gut (6 to 12 weeks) emphasizes the importance of GM in anti-HBV immunity. $^{25}$

Acute HBV infection can progress to chronic hepatitis $B$ in just $5 \%$ of adult patients, while the proportion in children is very different because more than $90 \%$ of exposed neonates and 30 to $50 \%$ of children aged 1 to 5 years fail to clear $\mathrm{HBV}^{8-10}$ It shows that adults have the highest rates of new infections and acute disease, but chronic infection is more likely to occur in infants or young children. It is due to their immune immaturity and unstable flora of the intestines. However, the adult epithelial cell surface is protected by the antimicrobial peptide, which can play an important role against the virus and experiencing an abrupt onset after HBV infection. ${ }^{21,26}$

It also implies a new therapeutic approach for patients with $\mathrm{HBV}$ infection. ${ }^{14}$ Fecal microbiota transplantation (FMT), in addition to standard antiviral, is effective in HBeAg clearance. ${ }^{27}$ Consequently, the frequency and production of chronic HBV infection depend not only on the direction of $\mathrm{HBV}$ invasion, virulence, immune system maturity, and viral load but also on the stability of GM.

\section{Gut Microbiota}

The gut microbiota (GM) is a complex ecosystem composed of a distinct array of bacteria that have a symbiotic relationship with each other and other microorganisms. From an immunological perspective, microorganisms are considered as pathogens by the host immune system that recognizes and destroys them. However, the immune system has coevolved to live in a collaborative relationship with the healthy microbiota. ${ }^{28}$

The GM colonizes the human intestinal tract and has a vital role in health and disease. The intestine of a healthy adult harbors 100 trillion microbial cells and has a complex genome of 150-fold more genes than the human genome and consists of 6-10 major phyla and 3000-5000 species. $^{29}$ Bacteroides and Firmicutes are the major phyla, followed by Proteobacteria and Actinobacteria, together representing more than $97 \%$ of the total microbiota. ${ }^{9}$

Gut microbiota can supplement the nutritional needs of the host by breaking down and absorbing complex dietary carbohydrates that cannot be digested by human enzymes, as well as synthesizing some essential substances, such as vitamins. ${ }^{30}$ Besides, it helps maintain the integrity of the intestinal epithelial barrier through the production of shortchain fatty acids, particularly butyrate, that play an important role in providing energy for cellular metabolism and regulating apoptosis, cellular differentiation, and chemical modification of nuclear proteins and nucleic acid. ${ }^{31}$

The role of gut microbiota in the regulation of immune cell homeostasis is increasingly recognized. Intestinal bacterial signals are responsible for priming systemic immune responses, including $\mathrm{T}$ regulatory cells (Tregs) and $\mathrm{T}$ helper 17 (Th17) cells, and for controlling pro- and anti-inflammatory host immune responses. Commensal species such as fragilis and Clostridial species promote Treg differentiation in the intestine. Furthermore, segmented filamentous bacteria are responsible for the induction of Th17 cytokines and initiate the maturation of natural killer $\mathrm{T}$ cells. ${ }^{14,32}$ Different genetic and environmental factors affect the composition of GM among individuals, but the human body can eventually develop a stable structure of intestinal flora and monitor the health of the body. ${ }^{33}$

Thus, the intestinal immune function cannot be neglected. In chronic $\mathrm{HBV}$-infected patients, gram-negative bacteria and inflammatory cascade were increased; however, anti-inflammatory and beneficial bacteria were depleted. In addition to this, the different studies also demonstrated that disruption of intestinal homeostasis 
and alterations in the intestinal microbiome contribute to a variety of diseases such as inflammatory bowel disease (IBD) and autoimmune liver disease. Moreover, the gut microbiota is implicated in non-alcoholic liver fat disease (NAFLD) and is associated with NAFLD progression to non-alcoholic hepatitis, cirrhosis, or hepatocellular carcinoma (HCC). ${ }^{34,35}$

\section{Gut-Liver Axis}

The "gut-liver axis" is defined as a close relationship between the gut and the liver because of its structural, functional, and bidirectional characteristics. The portal system gained approximately $75 \%$ blood from the gut, and intestinal blood content activates liver functions. The liver, in turn, affects intestinal functions through bile secretion into the intestinal lumen. ${ }^{14}$ The liver is the initial organ that is exposed to products originating from the gut, including not only the alimentary but also bacteria and their products. In healthy conditions, the protective mechanism for preserving hepatic immune homeostasis relies primarily on the roles of the intestinal barrier and the detoxifying ability of the liver. ${ }^{36}$

There is a profound impact on the immunological axis between the liver and intestine against microbe invasion. In other words, the interplay between the gut microbiota and liver pathology through the gut-liver axis plays an important role in determining the disease severity and prognosis of chronic HBV liver diseases. ${ }^{17}$

\section{Gut Microbiota in Chronic HBV Infection}

Now, there is emerging evidence that chronic liver disease has been related to imbalanced intestinal microbiota homeostasis, which has qualitative (dysbiosis) or quantitative (overgrowth) variations. ${ }^{29,37}$ Recently, some researchers are beginning to investigate the relationship between the diversity and composition of human gut microbiota and chronic hepatitis B virus infection. Chronic hepatitis $B$ virus infection exhibits alterations in the relative levels of "beneficial" and potentially "harmful" bacteria compared to healthy subjects; however, the dynamic alteration of the gut microbiota following HBV infection is still unknown. ${ }^{14,38,39}$

There are mutually causal structural changes in the intestinal microflora and the incidence of liver disease. The gut microbiota in chronic hepatitis B and liver cirrhosis patients have a reduced ratio of Bifidobacteriaceae/
Enterobacteriaceae (B/E), based on low levels of Bifidobacteria and Lactobacillus and high levels of Enterococcus and Enterobacteriaceae. The outcome suggested that the $\mathrm{B} / \mathrm{E}$ ratio is useful for following the extent of intestinal microecological disorder in the course of liver disease progression. ${ }^{1}$ In addition, these authors also found that the intestinal microflora composition of patients with CHB had changed compared to that of patients with chronic HBV infection before the serious liver injury, suggesting that changes in intestinal flora played a possible pathogenic role in patients with chronic HBV infection. ${ }^{1}$ A study reported that GM of patients with HBV-related cirrhosis contained lower levels of Bacteroidetes and increased levels of Enterobacteriaceae, Veillonellaceae and Streptococcaceae compared to the healthy group. ${ }^{39}$

Recent research has compared patients with asymptomatic carriage of hepatitis B virus (HBV), chronic hepatitis B (CHB), decompensated HBV cirrhosis, and healthy controls. Faecalibacterium prausnitzii, Enterococcus faecalis, and Enterobacteriaceae showed a marked increase in asymptomatic carriers compared to healthy controls, and the increased range was much greater in patients with chronic hepatitis B and those with decompensated HBV cirrhosis. The numbers of bifidobacteria and lactic acid bacteria (Lactobacillus, Pediococcus, Leuconostoc, and Weissella) dropped dramatically in both patient groups. In addition, gut permeability is altered in HBV-infected patients with an increase in bacterial translocation and endotoxin load in the portal vein that caused Toll-like receptor (TLR) activation in the liver, which facilitates immune-mediated liver injury. ${ }^{40,41}$

Constantly, functional gene array data showed that in both the HBV-related cirrhosis group and the alcoholic cirrhosis group, the abundance of functional genes important to nutrient metabolism, including amino acid metabolism, lipid metabolism, nucleotide metabolism, and isoprenoid biosynthesis, was significantly decreased compared to normal controls. ${ }^{42}$

In another study, patients with alcohol-related and HBV-related cirrhosis showed reduced GM diversity, predominantly Enterobacteriaceae and Streptococcaceae, compared to healthy individuals. ${ }^{43}$ Generally, the overgrowth of intestinal pathogenic bacteria leads to improved permeability of the mucosa, which facilitates immunemediated liver injury and the cause of systemic immune activation in chronic HBV infection. Therefore, these accumulating studies indicate that changes in gut 
microbiota composition play an important role in the induction and furthering the development of HBV-induced chronic liver disease. ${ }^{40}$

\section{Immune Function of Gut Microbiota in the Development of Chronic HBV Infection}

In recent years, different studies indicated that the gut microbiota composition influences the host immune response to $\mathrm{HBV}$, and when the intestinal flora is changed, the infection can easily turn into a chronic infection. ${ }^{25}$ Immune injuries triggered by structural changes in the gut microbiota are often caused by inflammatory pathways between the gut microbiota, the immune system, and the liver initiated by crosstalk. ${ }^{32}$

Kupffer cells, hepatic stellate cells, plasmacytoid dendritic cells (pDCs), and hepatocyte cells are capable of expressing TLRs that recognize pathogen-associated molecular patterns (PAMPs) and initiating the innate immune response. $^{44}$ Intestinal PAMPs associated with chronic infection with HBV are now considered to consist predominantly of lipopolysaccharide (LPS), bacterial DNA/ RNA, teichoic acid (TA), peptidoglycan (PGN) and nonmethylated $\mathrm{CpG}$ DNA, flagellin, and metabolic byproducts. $^{40}$

Lipopolysaccharide (LPS) is a critical component of gram-negative bacteria's outer membrane and is an endotoxin produced primarily by Enterobacteriaceae. Under normal physiological conditions, low concentrations of endotoxin entering the liver via the portal blood flow are almost completely scavenged by Kupffer cells since the liver is the key anti-endotoxin defense organ. ${ }^{45}$ However, in chronic $\mathrm{HBV}$-infected patients, pathogenic organisms produce a high amount of endotoxin. This lipopolysaccharide binds to LPS-binding protein, and TLR4 may recognize this combination on the surface of mononuclear macrophages. ${ }^{11}$ This recognition then stimulates CD14+ Kupffer cells, triggers the inflammatory cascade impact, activates the pathway associated with nuclear factor kappa $\mathrm{B}$ (NFforB), and produces inflammatory cytokines such as tumor necrosis factor- $\alpha$ (TNF- $\alpha$ ), IL-1, and IL- 6 and thus causes the development of liver fibrosis and cirrhosis. ${ }^{46,47}$

Another study showed that LPS can down-regulate the expression of tight junction proteins (closed proteins) in the intestinal tract, increase the permeability of the intestinal mucosa and reach the bloodstream via the portal venous system. $^{48}$ Additionally, TLR4 is also expressed by hepatic stellate cells and can release a large number of LPS-TLR4 pathway-dependent extracellular matrix proteins. In the fibrotic phase, these proteins are involved and may also be among the factors that cause chronic HBV infection to evolve into liver fibrosis. ${ }^{19}$ Evidence showed that the level of serum LPS in patients with chronic HBV liver failure is higher, which indicates that LPS may be related to the severity of the disease and a decreased clearance of endotoxin from the blood by the liver. ${ }^{16}$ Likewise, the implication of TLR4 in liver disease has also been proven, and it is one of the seven genes linked to an elevated risk of developing cirrhosis in patients with chronic hepatitis $\mathrm{C}$ infection. ${ }^{49}$

Other translocated bacterial products can also activate an inflammatory response by activating TLRs. TLR-2 recognize peptidoglycan (PGN) and lipoteichoic teichoic acid (TA), which are components of the cell wall of gram-positive bacteria, and TLR5 are mainly activated by bacterial flagellin, whereas TLR-9 is recognized as unmethylated CpG DNA, and TLR3 can combine with dsRNA in bacteria, and ssRNA can activate TLR7 and TLR8 receptors. ${ }^{50,51}$

The final downstream effect is the production of inflammatory cytokines, such as TNF-alpha, IL-1-beta, and IL-6, via the NF-kB pathway. However, if the immune response lasts for a long time or reaches an excessive intensity, this mechanism can also exacerbate liver damage, resulting in the release of a large number of cytokines in the body. ${ }^{50}$ Besides the gut microbiota is altered, the Th17 lymphocytes have been detected in the serum of chronic hepatitis $B$ patients. It is found occasionally in the liver and comes mostly from the gut. It is associated with poor prognosis, possibly due to abnormal secretion of IL-17A that increases angiogenesis and inflammatory cytokine production. ${ }^{52}$

Generally, alterations in gut microbiota and colonization by opportunistic bacteria can increase the risk of developing comorbid conditions in patients with HBVinduced chronic liver disease. Restructuring of those gut microbiota is a successful treatment for those patients.

\section{The Future Therapeutic Target for Chronic HBV Infection}

The first step in the development of a new treatment is to set targets such as reversal or prevention of fibrosis/cirrhosis progression, improvement or maintenance of liver homeostasis, or prevention of transplantation or death. Many different antiviral drugs are used in the treatment of chronic HBV infection, for various reasons, and it seems likely that chronic HBV infection will evolve into 
liver cirrhosis, liver failure, or liver cancer. Currently, a new therapeutic target has been established by studies on gut microbiota. ${ }^{11}$

Fecal microbiota transplantation (FMT) refers to the process of infusing fecal suspension from a healthy donor to the intestinal tract of a recipient to normalize the intestinal microbiota's composition and functionality. FMT can be applied to normalize the composition of the gut microbiota and increase the proportion and diversity of beneficial bacteria. FMT also provides the necessary signals for epithelial regeneration, induces mucins and antimicrobial peptide production, and reduces intestinal permeability to preserve the integrity of the epithelial barrier. ${ }^{53}$

Currently, FMT is emerging as one of the more hopeful treatments for chronic HBV infection. FMT has been conducted in HBeAg-positive chronic hepatitis B patients combined with ongoing entecavir (ETV) and tenofovir disoproxil fumarate (TDF) therapy, and $80 \%$ of them have reached $\mathrm{HBeAg}$ clearance. In contrast to the direct and indirect costs of repeated antiviral therapy courses, the cost of transplanting intestinal microbiota is bound to be lower. ${ }^{27}$

In addition to this, several reviews are summarizing the beneficial effect of probiotics on liver diseases. ${ }^{54-58}$ Probiotics are defined as live microorganisms that are ingested as food or medicinal supplements with beneficial effects on health. ${ }^{59,60}$ In chronic liver diseases, probiotics may improve the unhealthy state of the gut microbiota, as well as chronic inflammation. ${ }^{55}$ Sometimes, probiotics are species-specific or even strain-specific. ${ }^{61}$ Therefore, the option of strain or combination of strains is essential for therapeutic effectiveness.

Lactobacilli and bifidobacteria produce intestinal benefits that influence the immunity of the host, thus restoring gut dysbiosis, increasing the number of beneficial bacteria, and preserving the integrity of the intestinal barrier by preventing endotoxin translocation in chronically viralinfected patients. In this respect, it was found that antibacterial activity against common pathogenic bacteria is provided by Lactobacilli and bifidobacteria. ${ }^{62}$

\section{Conclusion}

Chronic hepatitis B virus infection is a global concern that affects human health. Some serious complications, such as liver failure, cirrhosis, and even hepatocellular carcinoma, may easily result from chronic HBV infection (HCC). Several studies have verified that there is a unique GM profile in chronic hepatitis B virus infection, closely correlated with immune responses and specific metabolic status. It increases intestinal permeability, impaired gut barrier, bacterial overgrowth, and translocation of bacteria, which facilitates liver injury mediated by immunity. Therefore, the role of gut microbiota should not be underestimated; rather careful attention to patients' intestinal micro-ecology should provide a sound basis for the treatment of chronic HBV infection. Many different antiviral drugs are used in the treatment of chronic HBV infection; for various reasons, it seems likely that chronic HBV infection well evolves into liver cirrhosis, liver failure, or liver cancer. So, we have been moving forwards with the use of FMT and probiotics. FMT is emerging as one of the most promising treatments for chronic HBV infection by manipulating human commensal bacteria. However, the available data in this field remains limited and further research is urgently needed. A detailed understanding of the mechanism of action between gut microbiota and HBV-related diseases is needed. It should be further studied in the future.

\section{Author Contributions}

All authors made substantial contributions to conception and design, acquisition of data, or analysis and interpretation of data; took part in drafting the article or revising it critically for important intellectual content; agreed to submit to the current journal; gave final approval of the version to be published; and agree to be accountable for all aspects of the work.

\section{Funding}

No funding sources.

\section{Disclosure}

The authors declare that they have no conflicts of interest.

\section{References}

1. Wang J, Wang Y, Zhang X, et al. Gut microbial dysbiosis is associated with altered hepatic functions and serum metabolites in chronic hepatitis B patients. Front Microbiol. 2017;8:2222. doi:10.3389/ fmicb.2017.02222

2. Rajoriya N, Combet C, Zoulim F, Janssen HL. How viral genetic variants and genotypes influence disease and treatment outcome of chronic hepatitis B. Time for an individualized approach? J Hepatol. 2017;67(6):1281-1297. doi:10.1016/j.jhep.2017.07.011

3. Gerlich WH. Medical virology of hepatitis B: how it began and where we are now. Virol J. 2013;10(1):239. doi:10.1186/1743-422X-10-239

4. Kramvis A. Genotypes and genetic variability of hepatitis B virus. Intervirology. 2014;57(3-4):141-150. doi:10.1159/000360947

5. Tong S, Revill P. Overview of hepatitis B viral replication and genetic variability. J Hepatol. 2016;64(1).

6. Peters MG. Hepatitis B virus infection: what is current and new. Top Antivir Med. 2019;26(4):112. 
7. Bonino F, Piratvisuth T, Brunetto MR, Liaw Y-F. Diagnostic markers of chronic hepatitis B infection and disease. Antivir Ther. 2010;15 (3):35. doi:10.3851/IMP1622

8. Pardee M. Diagnosis and management of hepatitis B and C. Nurs Clin. 2019;54(2):277-284. doi:10.1016/j.cnur.2019.02.004

9. Milosevic I, Vujovic A, Barac A, et al. Gut-liver axis, gut microbiota, and its modulation in the management of liver diseases: a review of the literature. Int J Mol Sci. 2019;20(2):395. doi:10.3390/ijms20020395

10. Yuen M-F, Ahn SH, Chen D-S, et al. Chronic hepatitis B virus infection. J Clin Gastroenterol. 2016;50(4):286-294. doi:10.1097/ MCG.0000000000000478

11. Yang R, Xu Y, Dai Z, Lin X, Wang H. The immunologic role of gut microbiota in patients with chronic HBV infection. J Immunol Res. 2018;2018:1-6. doi:10.1155/2018/2361963

12. Schnabl B, Brenner DA. Interactions between the intestinal microbiome and liver diseases. Gastroenterology. 2014;146(6):1513-1524. doi:10.1053/j.gastro.2014.01.020

13. Tsiaoussis GI, Assimakopoulos SF, Tsamandas AC, Triantos CK, Thomopoulos KC. Intestinal barrier dysfunction in cirrhosis: current concepts in pathophysiology and clinical implications. World J Hepatol. 2015;7(17):2058-2068. doi:10.4254/wjh.v7.i17.2058

14. Kang Y, Cai Y. Gut microbiota and hepatitis-B-virus-induced chronic liver disease: implications for fecal microbiota transplantation therapy. J Hospital Infect. 2017;96(4):342-348. doi:10.1016/j. jhin.2017.04.007

15. Organization WH. Global hepatitis report 2017: World Health Organization; 2017.

16. Kumar A, Kumar A. Innate immune responses in hepatitis B virus (HBV) infection. Virol J. 2014;11(1):22. doi:10.1186/1743-422X-1122

17. Xu D, Huang Y, Wang J. Gut microbiota modulate the immune effect against hepatitis B virus infection. Eur J Clin Microbiol Infect Dis. 2015;34(11):2139-2147. doi:10.1007/s10096-015-2464-0

18. Kawasaki T, Kawai T. Toll-like receptor signaling pathways. Front Immunol. 2014;5(461):1-8. doi:10.3389/fimmu.2014.00461

19. Zhang E, Lu M. Toll-like receptor (TLR)-mediated innate immune responses in the control of hepatitis B virus (HBV) infection. $\mathrm{Med}$ Microbiol Immunol. 2015;204(1):11-20. doi:10.1007/s00430-0140370-1

20. Ma Z, Zhang E, Yang D, Lu M. Contribution of Toll-like receptors to the control of hepatitis $B$ virus infection by initiating antiviral innate responses and promoting specific adaptive immune responses. Cell Mol Immunol. 2015;12(3):273. doi:10.1038/cmi.2014.112

21. Tsai K-N, Kuo C-F, Ou J-HJ. Mechanisms of hepatitis B virus persistence. Trends Microbiol. 2018;26(1):33-42. doi:10.1016/j. tim.2017.07.006

22. Sekirov I, Russell SL, Antunes LCM, Finlay BB. Gut microbiota in health and disease. Physiol Rev. 2010;90(3):859-904. doi:10.1152/ physrev.00045.2009

23. Mazagova M, Wang L, Anfora AT, et al. Commensal microbiota is hepatoprotective and prevents liver fibrosis in mice. FASEB J. 2014;29(3):1043-1055. doi:10.1096/fj.14-259515

24. Zhu Q, Xia P, Zhou X, et al. Hepatitis B virus infection alters gut microbiota composition in mice. Front Cell Infect Microbiol. 2019;9:377. doi:10.3389/fcimb.2019.00377

25. Chou -H-H, Chien W-H, Wu -L-L, et al. Age-related immune clearance of hepatitis $\mathrm{B}$ virus infection requires the establishment of gut microbiota. Proc Nat Acad Sci. 2015;112(7):2175-2180. doi:10.1073/ pnas. 1424775112

26. Korpela K, Salonen A, Virta LJ, et al. Intestinal microbiome is related to lifetime antibiotic use in Finnish pre-school children. Nat Commun. 2016;7:10410. doi:10.1038/ncomms10410

27. Ren YD, Ye ZS, Yang LZ, et al. Fecal microbiota transplantation induces hepatitis $\mathrm{B}$ virus e-antigen $(\mathrm{HBeAg})$ clearance in patients with positive $\mathrm{HBeAg}$ after long-term antiviral therapy. Hepatology. 2017;65(5):1765-1768. doi:10.1002/hep.29008
28. Bajaj JS. The role of microbiota in hepatic encephalopathy. Gut Microbes. 2014;5(3):397-403. doi:10.4161/gmic.28684

29 Chassaing B, Etienne-Mesmin L, Gewirtz AT. Microbiota-liver axis in hepatic disease. Hepatology. 2014;59(1):328-339. doi:10.1002/ hep. 26494

30. Minemura M, Shimizu Y. Gut microbiota and liver diseases. WJG. 2015;21(6):1691. doi:10.3748/wjg.v21.i6.1691

31. Bhat M, Arendt BM, Bhat V, Renner EL, Humar A, Allard JP. Implication of the intestinal microbiome in complications of cirrhosis. World J Hepatol. 2016;8(27):1128. doi:10.4254/wjh.v8. i27.1128

32. Liu Q, Li F, Zhuang Y, et al. Alteration in gut microbiota associated with hepatitis B and non-hepatitis virus related hepatocellular carcinoma. Gut Pathog. 2019;11(1):1. doi:10.1186/s13099-018-0281-6

33. Wang Y, Pan CQ, Xing H. Advances in gut microbiota of viral hepatitis cirrhosis. Biomed Res Int. 2019;2019.

34. Fukui H. Gut microbiota and host reaction in liver diseases. Microorganisms. 2015;3(4):759-791. doi:10.3390/ microorganisms3040759

35. Ponziani FR, Bhoori S, Castelli C, et al. Hepatocellular carcinoma is associated with gut microbiota profile and inflammation in nonalcoholic fatty liver disease. Hepatology. 2019;69(1):107-120. doi:10.1002/hep.30036

36. Lin R, Zhou L, Zhang J, Wang B. Abnormal intestinal permeability and microbiota in patients with autoimmune hepatitis. Int J Clin Exp Pathol. 2015;8(5):5153.

37. Schnabl B. Linking intestinal homeostasis and liver disease. Curr Opin Gastroenterol. 2013;29(3):264. doi:10.1097/ MOG.0b013e32835ff948

38. Fouts DE, Torralba M, Nelson KE, Brenner DA, Schnabl B. Bacterial translocation and changes in the intestinal microbiome in mouse models of liver disease. $J$ Hepatol. 2012;56(6):1283-1292. doi:10.1016/j.jhep.2012.01.019

39. Wei X, Yan X, Zou D, et al. Abnormal fecal microbiota community and functions in patients with hepatitis B liver cirrhosis as revealed by a metagenomic approach. BMC Gastroenterol. 2013;13(1):175. doi:10.1186/1471-230X-13-175

40. Lu H, Wu Z, Xu W, Yang J, Chen Y, Li L. Intestinal microbiota was assessed in cirrhotic patients with hepatitis B virus infection. Microb Ecol. 2011;61(3):693-703. doi:10.1007/s00248-010-9801-8

41. Yun Y, Chang Y, Kim H-N, et al. Alterations of the gut microbiome in chronic hepatitis B virus infection associated with alanine aminotransferase level. J Clin Med. 2019;8(2):173. doi:10.3390/ jcm8020173

42. Chen Y, Qin N, Guo J, et al. Functional gene array-based analysis of fecal microbiomes in patients with liver cirrhosis. BMC Genom. 2014;15(1):753. doi:10.1186/1471-2164-15-753

43. Chen Y, Yang F, Lu H, et al. Characterization of fecal microbial communities in patients with liver cirrhosis. Hepatology. 2011;54 (2):562-572. doi:10.1002/hep.24423

44. Chen J, Wei Y, He J, et al. Natural killer T cells play a necessary role in modulating immune-mediated liver injury by gut microbiota. Sci Rep. 2014;4:7259. doi:10.1038/srep07259

45. Cinar MU, Islam MA, Pröll M, et al. Evaluation of suitable reference genes for gene expression studies in porcine PBMCs in response to LPS and LTA. BMC Res Notes. 2013;6(1):56. doi:10.1186/17560500-6-56

46. Bermudez-Brito M, Muñoz-Quezada S, Gomez-Llorente C, et al. Cell-free culture supernatant of Bifidobacterium breve CNCM I-4035 decreases pro-inflammatory cytokines in human dendritic cells challenged with Salmonella typhi through TLR activation. PLoS One. 2013;8(3):e59370. doi:10.1371/journal.pone.0059370

47. Seki E, Schnabl B. Role of innate immunity and the microbiota in liver fibrosis: crosstalk between the liver and gut. J Physiol. 2012;590 (3):447-458. doi:10.1113/jphysiol.2011.219691 
48. Frazier TH, DiBaise JK, McClain CJ. Gut microbiota, intestinal permeability, obesity-induced inflammation, and liver injury. $J$ Parenteral Enteral Nutr. 2011;35:14S-20S. doi:10.1177/ 0148607111413772

49. Allam NG, Salem ML, Elbatae H, Nabieh MM. Lactobacillus acidophilus and Bifidobacteria spp having antibacterial and antiviral effects on chronic HCV infection. African Journal of Microbiology Research. 2019;13(5):77-90.

50. Luedde T, Schwabe RF. NF- $\mathrm{BB}$ in the liver-linking injury, fibrosis, and hepatocellular carcinoma. Nat Rev Gastroenterol Hepatol. 2011;8 (2):108. doi:10.1038/nrgastro.2010.213

51. Kawai T, Akira S. The role of pattern-recognition receptors in innate immunity: update on Toll-like receptors. Nat Immunol. 2010;11 (5):373. doi:10.1038/ni.1863

52. Ponziani FR, Nicoletti A, Gasbarrini A, Pompili M. Diagnostic and therapeutic potential of the gut microbiota in patients with early hepatocellular carcinoma. Ther Adv Med Oncol. 2019;11:1754. doi:10.1177/1758835919848184

53. Taur Y, Coyte K, Schluter J, et al. Reconstitution of the gut microbiota of antibiotic-treated patients by autologous fecal microbiota transplant. Sci Transl Med. 2018;10(460):9489. doi:10.1126/scitranslmed.aap9489

54. Cesaro C, Tiso A, Del Prete A, et al. Gut microbiota and probiotics in chronic liver diseases. Digestive Liver Dis. 2011;43(6):431-438. doi:10.1016/j.dld.2010.10.015
55. Kirpich IA, McClain CJ. Probiotics in the treatment of liver diseases. J Am Coll Nutr. 2012;31(1):14-23. doi:10.1080/ 07315724.2012 .10720004

56. Nitin J, Mithun S, Rao P, Nageshwar Reddy D. Liver diseases: the role of gut microbiota and probiotics. J Prob Health. 2016;4(154):2. doi:10.4172/2329-8901.1000154

57. Elzouki A-N. Probiotics and liver disease: where are we now and where are we going? J Clin Gastroenterol. 2016;50:188-190. doi:10.1097/MCG.0000000000000712

58. Sharma V, Garg S, Aggarwal S. Probiotics and liver disease. Perm J. 2013;17(4):62. doi:10.7812/TPP/12-144

59. Butel M-J. Probiotics, gut microbiota and health. Médecine et maladies infectieuses. 2014;44(1):1-8. doi:10.1016/j.medmal.2013.10.002

60. Ganguly N, Bhattacharya S, Sesikeran B, et al. ICMR-DBT guidelines for evaluation of probiotics in food. Indian J Med Res. 2011;134 (1):22.

61. Gibson GR, Hutkins R, Sanders ME, et al. Expert consensus document: the International Scientific Association for Probiotics and Prebiotics (ISAPP) consensus statement on the definition and scope of prebiotics. Nat Rev Gastroenterol Hepatol. 2017;14(8):491. doi:10.1038/nrgastro.2017.75

62. Chen $\mathrm{C}$, Li L, Wu Z, Chen H, Fu S. Effects of lactitol on intestinal microflora and plasma endotoxin in patients with chronic viral hepatitis. J Infect. 2007;54(1):98-102. doi:10.1016/j.jinf.2005.11.013
Infection and Drug Resistance

\section{Publish your work in this journal}

Infection and Drug Resistance is an international, peer-reviewed openaccess journal that focuses on the optimal treatment of infection (bacterial, fungal and viral) and the development and institution of preventive strategies to minimize the development and spread of resistance. The journal is specifically concerned with the epidemiology of

\section{Dovepress}

antibiotic resistance and the mechanisms of resistance development and diffusion in both hospitals and the community. The manuscript management system is completely online and includes a very quick and fair peerreview system, which is all easy to use. Visit http://www.dovepress.com/ testimonials.php to read real quotes from published authors. 